\title{
Article \\ Estimation of the Frequency Response Function of the Rotational Degree of Freedom
}

\author{
Ji-wook Kim, Jae-wook Lee, Kun-woo Kim, Ji-heon Kang (D), Min-seok Yang, Dong-yul Kim, Seung-yeop Lee \\ and Jin-seok Jang *(D)
}

Citation: Kim, J.-w.; Lee, J.-w.; Kim, K.-w.; Kang, J.-h.; Yang, M.-s.; Kim,

D.-y.; Lee, S.-y.; Jang, J.-s. Estimation of the Frequency Response Function of the Rotational Degree of Freedom. Appl. Sci. 2021, 11, 8527. https:// doi.org/10.3390/app11188527

Academic Editor: Giuseppe Lacidogna

Received: 12 August 2021

Accepted: 12 September 2021

Published: 14 September 2021

Publisher's Note: MDPI stays neutral with regard to jurisdictional claims in published maps and institutional affiliations.

Copyright: (c) 2021 by the authors. Licensee MDPI, Basel, Switzerland. This article is an open access article distributed under the terms and conditions of the Creative Commons Attribution (CC BY) license (https:/ / creativecommons.org/licenses/by/ $4.0 /)$.
Smart Manufacturing Technology R\&D Group, Korea Institute of Industrial Technology, Daegu 42994, Korea; jwkim0@kitech.re.kr (J.-w.K.); jaewk@kitech.re.kr (J.-w.L.); kwkim@kitech.re.kr (K.-w.K.); kangji1226@kitech.re.kr (J.-h.K.); msyang@kitech.re.kr (M.-s.Y.); dyk87@kitech.re.kr (D.-y.K.); sylee94@kitech.re.kr (S.-y.L.)

* Correspondence: jsjang@kitech.re.kr; Tel.: +82-53-580-0152

\begin{abstract}
One of the factors that influence the dynamic characteristics of machining systems is the cutting tool. Cutting tools are very diverse, and receptance coupling substructure analysis (RCSA) is essential for analyzing the dynamic characteristics of each tool. For RCSA, a full receptance matrix of the equipment and tools is essential. In this study, rotational degree-of-freedom receptance was estimated and analyzed using translational receptance. Displacement/moment receptance was analyzed according to the distance of the response point using the first-and second-order finite difference methods. The rotation/moment receptance was estimated according to the distance of the response point. Rotation/moment receptance was analyzed using Schmitz's method and compensation strategies. The limitations of these strategies were analyzed, and the rotation/moment receptance for the beam under free-free boundary conditions was predicted using the second compensation strategy.
\end{abstract}

Keywords: receptance coupling substructure analysis; rotational FRF; finite difference technique; modal analysis

\section{Introduction}

To optimize the machining process and respond to abnormal conditions, it is essential to analyze chatter stability using the dynamic characteristics of the equipment and cutting tools. A chatter stability lobe diagram is utilized for chatter stability analysis, and an essential parameter is the dynamic characteristic of the machining system. The dynamic characteristics of machining are significantly affected by the equipment, material, and process characteristics, and the dynamic characteristics change as the cutting tool changes. The types of cutting tools utilized in processing equipment are very diverse, and it is very inefficient to change the cutting tools to analyze the dynamic characteristics of various cutting tools, perform vibration tests of processing equipment, and obtain dynamic characteristics. Receptance coupling substructure analysis (RCSA) decomposes a complex structure into relatively simple substructures and obtains the frequency response function (FRF) of the substructures using experiments or FEM. Subsequently, by synthesizing the FRF of the substructure, the FRF of the entire system is obtained, and the dynamic characteristics are predicted. Regarding a machining center, the FRF of the entire machining equipment system is obtained by synthesizing the FRFs of the cutting tool and machining equipment, and the dynamic characteristics are predicted. Through the RCSA of various cutting tools, the FRF and dynamic characteristics of the equipment can be predicted. It is more efficient in terms of time and cost to change solely the cutting tool and perform a vibration test rather than change the cutting tool repeatedly and perform a vibration test on the entire machining equipment [1-6].

To perform RCSA, the FRF for translational and rotational degrees of freedom of the substructure is required. In previous studies, errors occurred when RCSA was performed 
using only the translational FRF. When RCSA is performed using FRF in the rotational direction, the accuracy is improved [2-4,6]. However, it is difficult to obtain the rotational displacement and moment during the actual vibration test, and there is a disadvantage, in that the cost of additional experimental equipment is incurred. Rotational FRF has been known as a problem since 1972, as can be seen in the paper of Ewins and Sainsbury, and many studies to estimate accurate rotational FRF are still in progress [5,7]. Although FEM can be achieved without major problems, finding rotational FRF through practical experiments is rarely achieved. Experimentally, the block, mass additive, finite difference, estimation, angular transducer, and laser techniques have been used [5-10].

In this study, the problem was analyzed from an experimental point of view. Experimentally, the finite-difference method is used, which is low in cost and relatively simple to obtain accurate results, does not require additional equipment, and performs post-processing using translational FRF. When estimating the rotational FRF using the finite difference method, it is difficult to select the order of the finite difference method and the distance of the response point. In previous studies, only the order of the finite difference method was studied. In addition, there is only the suggestion of the distance of the measurement point without considering the size of the structure and the size of the sensor [6]. However, in this study, a suitable ratio of the measuring point distance to the size of the structure and the maximum measuring distance considering the sensor size during actual testing are presented. The FRF of rotational degrees of freedom was estimated and analyzed using the FRF of translational degrees of freedom according to the response distance [6-9]. To estimate and verify the rotational FRF, a vibration analysis was performed using Abaqus, a commercial program. To obtain the rotation/force and displacement/moment FRF, the rotational FRF according to the measurement distance was estimated, and the optimal condition was selected. In addition, the rotation/moment FRF is estimated and analyzed using Schmitz's method and a compensation strategy using modal parameters [11-13].

\section{Theoretical Background}

\subsection{Receptance of Euler-Bernoulli Beam}

The coordinates and directions of the displacement, rotation, force, and moment of the substructure are illustrated in Figure 1. The $i$ and $j$ at the ends indicate the measurement and excitation positions, respectively. In this study, as illustrated in Figure 1, the outof-plane mode is considered solely, and the axial direction is not considered. The full receptance matrix according to the RCSA formula can be expressed as:

$$
R_{i j}=\left[\begin{array}{ll}
H_{i j} & L_{i j} \\
N_{i j} & P_{i j}
\end{array}\right]=\left[\begin{array}{cc}
x_{i} / F_{j} & x_{i} / M_{j} \\
\theta_{i} / F_{j} & \theta_{i} / M_{j}
\end{array}\right]
$$

where $H_{i j}$ is the displacement/force FRF, $L_{i j}$ is the displacement/moment FRF, $N_{i j}$ is the rotation/force FRF, and $P_{i j}$ is the rotation/moment FRF.

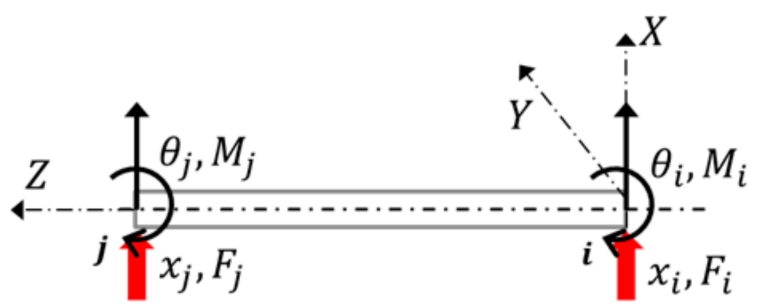

Figure 1. Coordinate system of the beam. 
In the free-free boundary condition of the beam, the cylindrical direct and cross receptance can be expressed using the Euler-Bernoulli beam theory, as expressed in Equations (2) and (3), respectively [14-17]:

$$
\begin{gathered}
R_{i j}=\left[\begin{array}{ll}
H_{i i} & L_{i i} \\
N_{i i} & P_{i i}
\end{array}\right]=\left[\begin{array}{cc}
\frac{-C_{1}}{E I \lambda^{3} C_{7}} & \frac{C_{2}}{E I \lambda^{2} C_{7}} \\
\frac{C_{2}}{E I \lambda^{2} C_{7}} & \frac{C_{3}}{E I \lambda C_{7}}
\end{array}\right] \\
R_{i j}=\left[\begin{array}{ll}
H_{i j} & L_{i j} \\
N_{i j} & P_{i j}
\end{array}\right]=\left[\begin{array}{cc}
\frac{C_{4}}{E I \lambda^{3} C_{7}} & \frac{-C_{5}}{E I \lambda^{2} C_{7}} \\
\frac{C_{5}}{E I \lambda^{2} C_{7}} & \frac{C_{6}}{E I \lambda C_{7}}
\end{array}\right]
\end{gathered}
$$

where $E$ is the elastic modulus, $I$ is the area moment of inertia, $\eta$ is the damping ratio, $L$ is the length of the cylinder, and $\lambda$ can be expressed as Equation (4).

$$
\lambda^{4}=\frac{\omega m}{E I(1+i \eta) L}
$$

where, $\left\{\begin{array}{c}C_{1}=\cos \lambda L \sinh \lambda L-\sin \lambda L \cosh \lambda L \\ C_{2}=\sin \lambda L \sinh \lambda L \\ C_{3}=\cos \lambda L \sinh \lambda L+\sin \lambda L \cosh \lambda L \\ C_{4}=\sin \lambda L-\sinh \lambda L \\ C_{5}=\cos \lambda L-\cosh \lambda L \\ C_{6}=\sin \lambda L+\sinh \lambda L \\ C_{7}=\cos \lambda L \cosh \lambda L-1\end{array}\right.$.

\subsection{Rotation/Force and Displacement/Moment FRF}

The rotation/force $(\mathrm{N})$ and displacement/moment (L) FRFs can be estimated using two or three translational FRFs measured at a constant distance, as illustrated in Figure 2. Rotational FRF can be estimated using the measured translational FRF transformation matrix, as expressed in Equation (5):

$$
\left[H_{\text {estimation }}\right]=[T]\left[H_{\text {Measurment }}\right][T]^{T}
$$

where the measured translational FRF is shown as Equation (6):

$$
\left[H_{\text {Measurment }}\right]=\left[\begin{array}{ccc}
H_{1 b 1 b} & H_{1 b 1 a} & H_{1 b 1} \\
H_{1 a 1 b} & {\left[\begin{array}{cc}
H_{1 a 1 a} & H_{1 a 1} \\
H_{11 a} & H_{11}
\end{array}\right]}
\end{array}\right]
$$

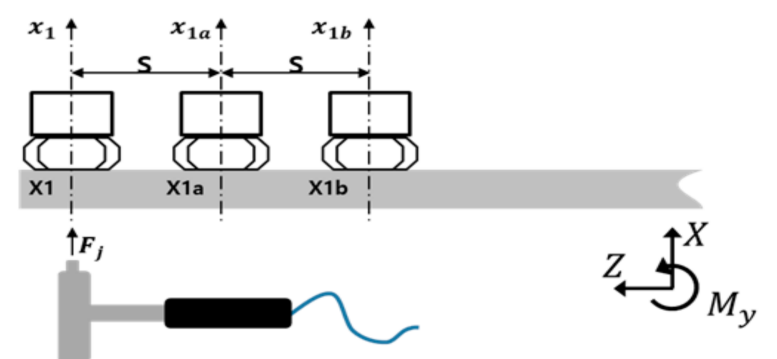

Figure 2. Impact and response point for rotational D.O.F.

The rotation transformation matrix using the finite difference method is obtained using Lagrange interpolation. When the domain for Lagrange interpolation in the experimental boundary is $(a, b)$ and the measurement point $x_{i}$ satisfies $a \leq x_{i} \leq b(i=0,1,2 \cdots n-1)$, the two-dimensional mode shape is as follows by Lagrange polynomials $[7,18-20]$ : 


$$
y(x)=\sum_{i=0}^{n-1} y_{i} a_{i}(x)
$$

where, $a_{i}(x)=\prod_{k \neq i}^{n-1} \frac{x-x_{k}}{x_{i}-x_{k}}, i=0,1,2, \cdots, n-1$.

The rotation matrix differentiates Equation (7) and is as follows:

$$
\delta_{b r}(x)=y^{\prime}(x)=\sum_{i=0}^{n-1} y_{i} a_{i}^{\prime}(x)
$$

Table 1 shows the results of the 2nd and 3rd interpolation. Using this interpolation formula, it is possible to obtain a transformation matrix $(\mathrm{T})$ that calculates the rotational freedom of the coupling region from the translational degrees of freedom in each direction at the nodes near the coupling region.

Table 1. Lagrange interpolation.

\begin{tabular}{cc}
\hline Order of Equation & $y^{\prime}$ \\
\hline 2nd order : $y=a_{2} x^{2}+a_{1} x+a_{0}$ & $\left(-3 y_{0}+4 y_{1}-y_{2}\right) / 2 h$ \\
\hline 3rd order : $y=a_{3} x^{3}+a_{2} x^{2}+a_{1} x+a_{0}$ & $\left(-11 y_{0}+18 y_{1}-9_{y 2}+2 y_{3}\right) / 6 h$ \\
\hline
\end{tabular}

When the third order or higher is reached, the FRF of the translational degrees of freedom to be measured increases, which may increase the experimental error. For this reason, only the first and second interpolation methods are used in this study. The transformation matrix $(T)$ using the finite difference method has first-order and second-order approximations, and the first-order requires two measurement points. Depending on the position of the rotational FRF to be estimated, forward and backward difference transformation matrices are adopted, as expressed in Equations (9) and (10), respectively.

$$
\begin{aligned}
& {\left[T_{1 f}\right]=\left[\begin{array}{cc}
0 & 1 \\
1 / s & -1 / s
\end{array}\right]} \\
& {\left[T_{1 b}\right]=\left[\begin{array}{cc}
0 & 1 \\
-1 / s & 1 / s
\end{array}\right]}
\end{aligned}
$$

The second order requires three measurement points, and they include the forward, central, and backward difference transformation matrices. Equations (11)-(13) are similar.

$$
\begin{aligned}
& {\left[T_{2 f}\right]=\frac{1}{2 s}\left[\begin{array}{ccc}
0 & 0 & 2 s \\
-1 & 4 & -3
\end{array}\right]} \\
& {\left[T_{2 c}\right]=\frac{1}{2 s}\left[\begin{array}{ccc}
0 & 2 s & 0 \\
-1 & 0 & 1
\end{array}\right]} \\
& {\left[T_{2 b}\right]=\frac{1}{2 s}\left[\begin{array}{ccc}
0 & 0 & 2 s \\
1 & -4 & 3
\end{array}\right]}
\end{aligned}
$$

In each transformation matrix, the forward, central, and backward parameters are related to the position to obtain the rotational FRF [11-16].

\subsection{Rotation/Moment FRF}

Regarding rotation/moment FRF (P), it can be obtained using Equation (5) in Section 2.2. In the finite difference method, the transformation matrix must be applied by determining the excitation and response for each of the three points. The experimental data yielded nine FRFs with three points of excitation, and three points of response for each point. When the 
finite difference method is adopted for rotation/moment FRF estimation, the number of experiments increase, which has a disadvantage in that it may increase the experimental error. To reduce the experimental error, it was estimated using Schmitz's method. Schmitz's method reduces experimental errors by post-processing of measured and calculated FRFs without requiring additional experiments. Regarding Schmitz's method, it can be expressed as Equation (14), and there is a disadvantage that the error increases when the anti-resonance of $H$ is approximately zero $[3,11,13]$.

$$
P_{11-\text { schmitz }}=\frac{\theta_{1}}{M_{1}}=\frac{L_{11}^{2}}{H_{11}}
$$

To remove the residual mode, a compensation strategy was proposed by Yulei Ji et al. [6]. Equation (15) represents the FRF using the mode superposition method; accordingly, $H_{11}$ and $L_{11}$ can be expressed as Equation (16).

$$
\begin{gathered}
\Phi=\sum_{i=1}^{2} \frac{1}{k_{i}}\left(\frac{1}{1-\left(\omega / \omega_{n, i}\right)^{2}+j 2 \zeta\left(\omega / \omega_{n, i}\right)}\right)=\sum_{i=1}^{2} \frac{1}{k_{i}} \times S_{i} \\
\left\{\begin{array}{c}
H_{11}=\sum_{i=1}^{2} k_{i, H_{11}}^{-1} \times S_{i}=k_{1, H_{11}}^{-1} \times S_{1}+k_{2, H_{11}}^{-1} \times S_{2} \\
L_{11}=\sum_{i=1}^{2} k_{i, L_{11}}^{-1} \times S_{i}=k_{1, L_{11}}^{-1} \times S_{1}+k_{2, L_{11}}^{-1} \times S_{2}
\end{array}\right.
\end{gathered}
$$

By substituting Equation (16) into Equation (15) and rearranging it, it can be expressed as Equation (17). In Equation (17), the first and second components represent the first and second modes, respectively, and the third mode represents the residual mode [11].

$$
P_{11}=\frac{k_{1, L_{11}}^{-1}}{k_{1, H_{11}}^{-1}} k_{1, L_{11}}^{-1} \times S_{1}+\frac{k_{2, L_{11}}^{-1}}{k_{2, H_{11}}^{-1}} k_{2, L_{11}}^{-1} \times S_{2}+K \frac{\prod k_{i, H_{11}}^{-1} \times S_{i}}{\sum k_{i, H_{11}}^{-1} \times S_{i}}
$$

where $K=\left(\frac{k_{2, L_{11}}^{-1}}{k_{2, H_{11}}^{-1}}\right)^{2}-\left(\frac{k_{1, L_{11}}^{-1}}{k_{1, H_{11}}^{-1}}\right)^{2}$.

An error occurs because of the third residual term, and if modes are expressed by eliminating the residual mode, two modes are as denoted in Equation (18).

$$
\left\{\begin{array}{l}
\text { Mode } 1=\frac{k_{1, L_{11}}^{-1}}{k_{1, H_{11}}^{-1}} k_{1, L_{11}}^{-1} \times S_{1} \\
\text { Mode } 2=\frac{k_{2, L_{11}}^{-1}}{k_{2, H_{11}}^{-1} k_{2, L_{11}}^{-1} \times S_{2}}
\end{array}\right.
$$

Similarly, there is a method for discarding residual modes by initially calculating Schmitz's method in Equation (14), and then performing mode analysis. Two compensation strategies are illustrated in Figure 3, and the first compensation strategy initially performs a mode analysis of $H_{11}$ and $L_{11}$ to calculate the rotation/moment FRF. The second compensation strategy eliminates the residual mode through mode analysis by initially performing Schmitz's method. The compensation strategy has the advantage of increasing the accuracy of $P_{11}$ by eliminating the residual mode in Schmitz's method and does not require additional experiments as a post-processing operation $[11,13]$. 


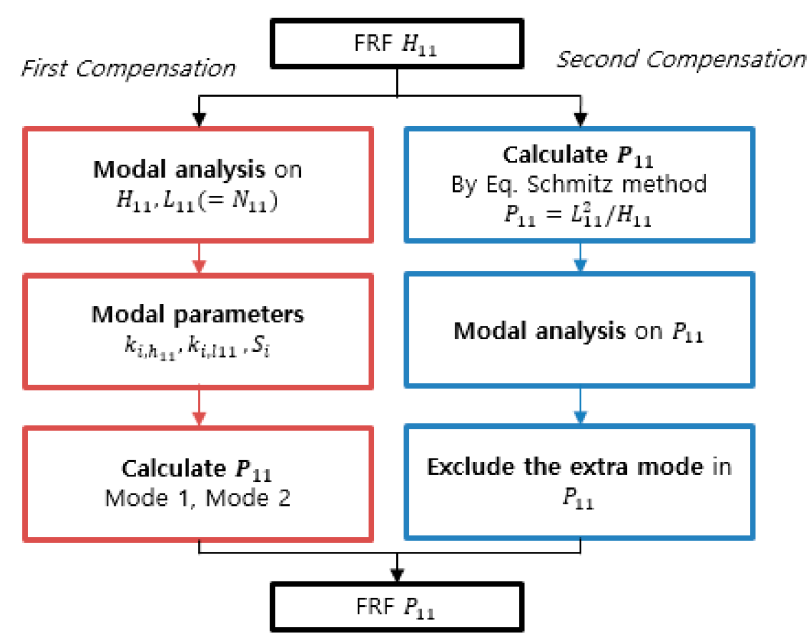

Figure 3. Two types of compensation strategy.

\subsection{Modal Peak Picking}

Modal analysis must be performed to conduct a compensation strategy. Modal analysis includes modal circle and modal peak picking. In this study, modal analysis was performed using modal peak picking. Modal peak picking is a method for obtaining modal parameters using real and imaginary numbers. Each number in Figure 4 represents the frequency value at the peak of the real and imaginary parts, and $A$ and $B$ represent the magnitude of the frequency in the imaginary part. The damping ratio, stiffness, mass, and damping coefficient are as denoted in Equations (19)-(22) [14-16].

$$
\begin{gathered}
\zeta_{q 1}=\frac{\omega_{4}-\omega_{3}}{2 \omega_{n 1}}, \quad \zeta_{q 2}=\frac{\omega_{6}-\omega_{5}}{\omega_{n 2}} \\
k_{q 1}=\frac{-1}{2 \zeta_{q 1} A}, \quad k_{q 2}=\frac{-1}{2 \zeta_{q 2} B} \\
m_{q 1}=\frac{k_{q 1}}{\omega_{n 1}^{2}}, \quad m_{q 2}=\frac{k_{q 1}}{\omega_{n 2}^{2}} \\
c_{q 1}=2 \zeta_{q 1} \sqrt{k_{q 1} m_{q 1}}, \quad c_{q 2}=2 \zeta_{q 2} \sqrt{k_{q 2} m_{q 2}}
\end{gathered}
$$
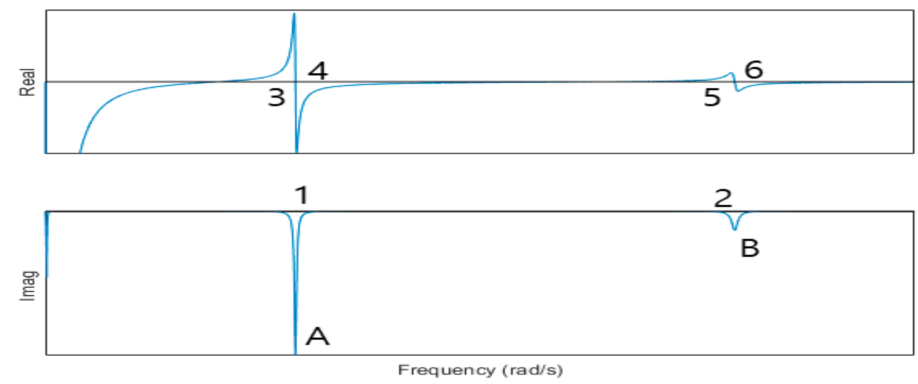

Figure 4. Modal peak picking method.

\section{Vibration Analysis Using FEM}

\subsection{FE Modeling}

In this study, the accuracy of the estimation of rotational FRFs according to the measurement distance and finite difference order are compared. In addition, the rotation/moment FRF estimation accuracy by the compensation strategy was compared, and the accuracy according to the boundary condition was analyzed. 
Two or three response points were required to estimate $L_{11}$ and $N_{11}$. It is difficult to obtain and verify the rotational degree of freedom FRF through an experiment. Therefore, CAE was adopted to compare the rotational FRF estimation accuracy according to the distance (s) of the response point. In modal analysis using CAE, in order to reduce the effect error on the higher-order mode, the analysis should be performed considering at least 10 times the mode of interest or more. To solely consider the out-of-plane mode, modeling is performed using the B22 element model, which is a beam element of the 2D planar. The material properties of the beam model adopted for the vibration analysis are listed in Table 2, and the boundary condition is a free-free condition. To compare the accuracy according to the measurement distance, the response was analyzed by dividing the ratio of the total length of the beam. Figure 5 illustrates the position of the response point, which is the point for obtaining the rotational FRF. When the ratio of the distance of the measuring point to the total length of the beam is $\mathrm{s} / 1, \mathrm{X} 01$ represents $\mathrm{s} / 1=0.01$. As such, $\mathrm{X} 05, \mathrm{X} 1$, $\mathrm{X} 2, \mathrm{X} 3, \mathrm{X} 4$, and $\mathrm{X} 5$ have $\mathrm{s} / 1$ of $0.05,0.1,0.2,0.3,0.4$, and 0.5 , respectively. To verify the reliability of the vibration analysis of FEM, the FEM at some points and Euler-Bernoulli beam FRF results are compared. As illustrated in Figure 6, FEM modeling was verified as the results of the FEM and the theory are consistent.

Table 2. Properties of the beam model.

\begin{tabular}{ccccc}
\hline Length & Diameter & Density & Elastic Modulus & Damping Ratio \\
\hline $1 \mathrm{~m}$ & $0.005 \mathrm{~m}$ & $7850 \mathrm{~kg} / \mathrm{m}^{3}$ & $210 \mathrm{GPa}$ & 0.01 \\
\hline
\end{tabular}

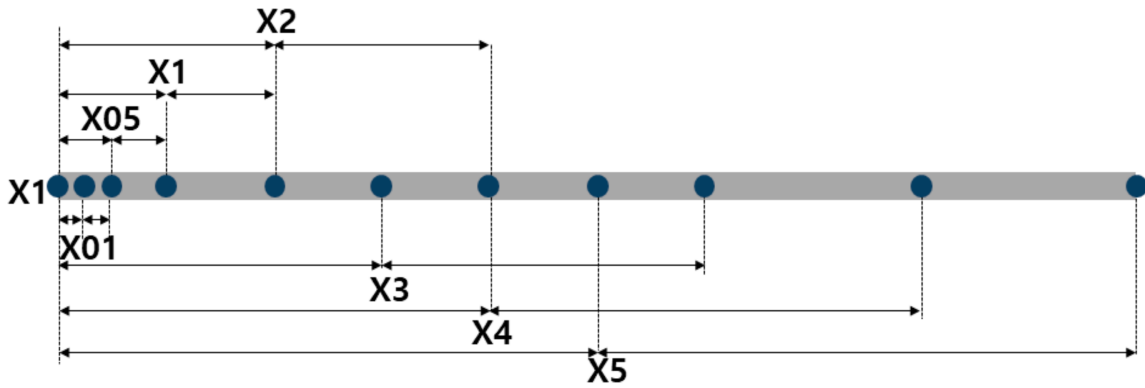

Figure 5. Response point of the beam model.

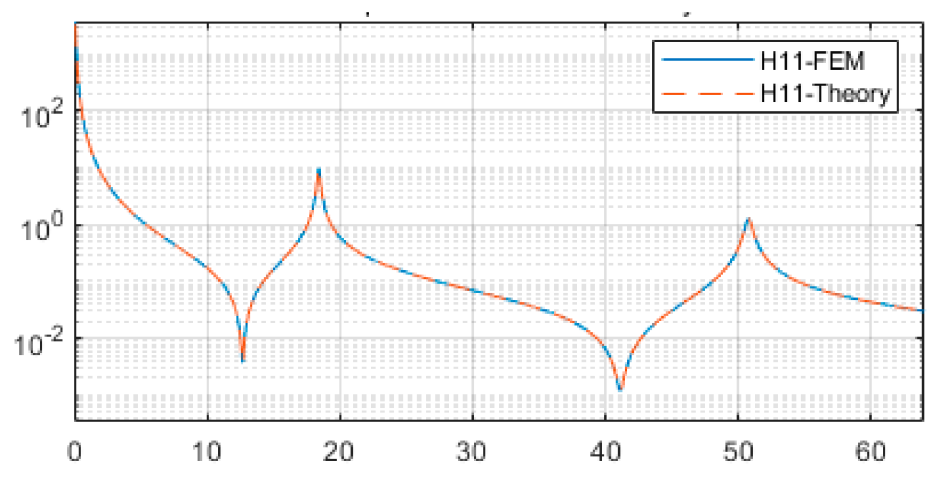

Figure 6. Verification of FEM and theory.

\subsection{Rotaion/Force(=Displacement/Moment) Vibration Analysis Results}

The estimated FRF, $L_{11}\left(=N_{11}\right)$, using the first-order finite difference method according to the response point distance, is illustrated in Figure 7. The natural frequency coincided with the FEM results regardless of the distance between the response points, but the magnitude of the natural frequency tended to decrease as the distance between the response points increased. The anti-resonance also exhibited a tendency to move as the distance of the response point increased. 


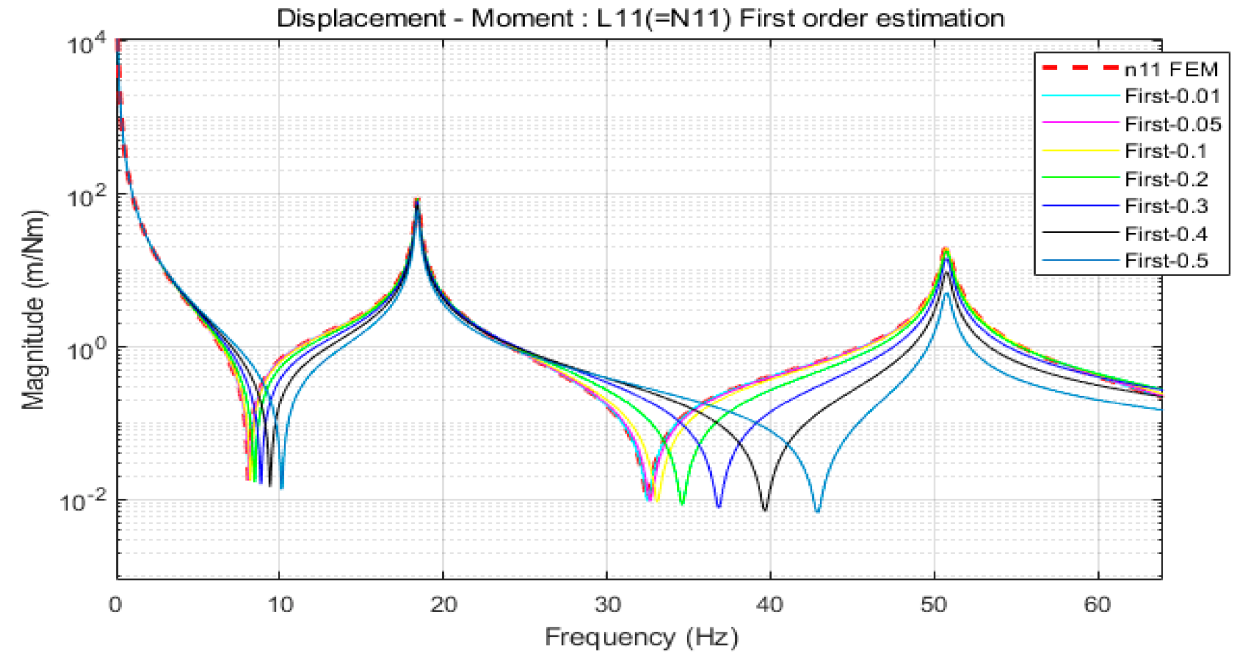

Figure 7. Estimation for $L_{11}\left(=N_{11}\right)$ for the first-order finite difference method.

The estimated FRF, $L_{11}\left(=N_{11}\right)$, using the second-order finite difference method according to the response point distance, is illustrated in Figure 8. Similar to the first-order method, the natural frequency coincided with the FEM result, and an error occurred in the natural frequency magnitude and anti-resonance. As the distance between the response points increased, the error increased.

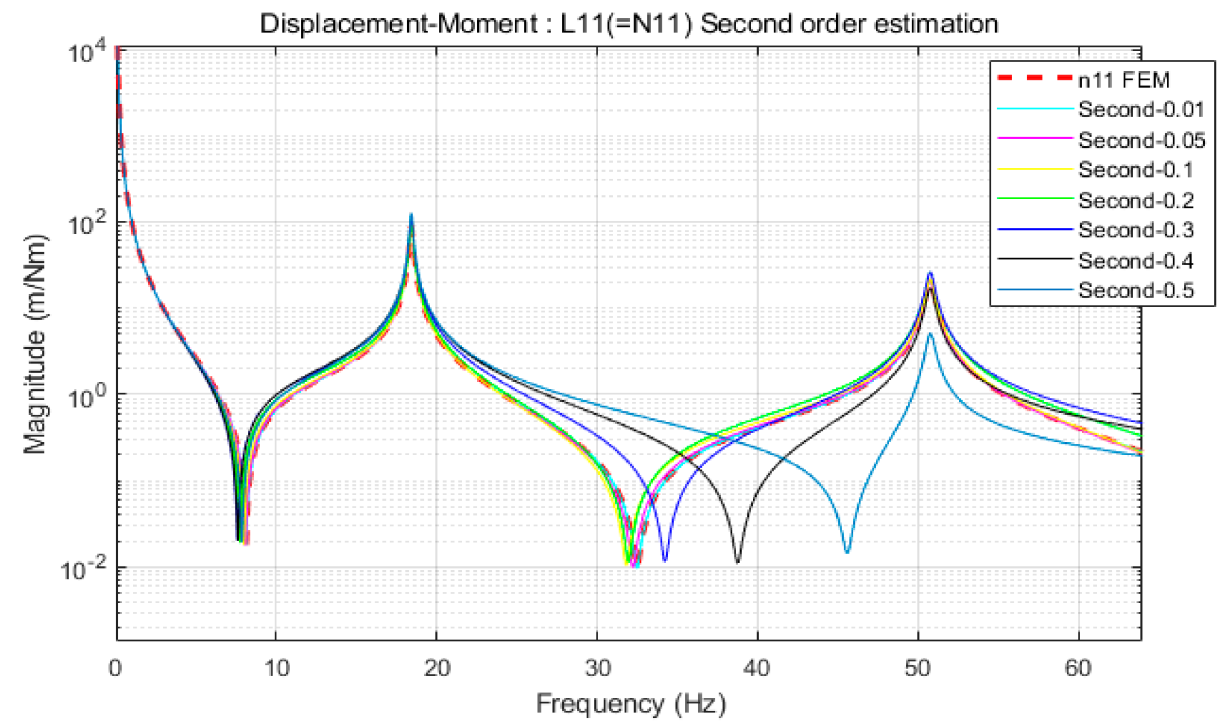

Figure 8. Estimation for $L_{11}\left(=N_{11}\right)$ for the second-order finite difference method.

The error rate for the first natural frequency magnitude of the FEM and the estimated FRF of the first-and second-order finite difference methods are illustrated in Figure 9. In both the first and second order, the error rate increased as the distance of the response point increased. When $\mathrm{s} / 1$ was 0.1 , the error rate of the first order was $0.4 \%$, and the second order was $2.1 \%$. When s/ 1 was 0.2 , the error rate of the first order was $2.9 \%$, and the second order was $12.3 \%$. The magnitude error increased as the higher-order mode goes. The estimation of $L_{11}\left(=N_{11}\right)$ using the finite difference method yielded more accurate results in the first order, and the shorter the distance between the response points, the more accurate the results. The ratio of the response point distance to the total length of the structure during the experiment should not exceed 0.05. Even considering the structure and sensor size, it should not exceed 0.1 at most. 


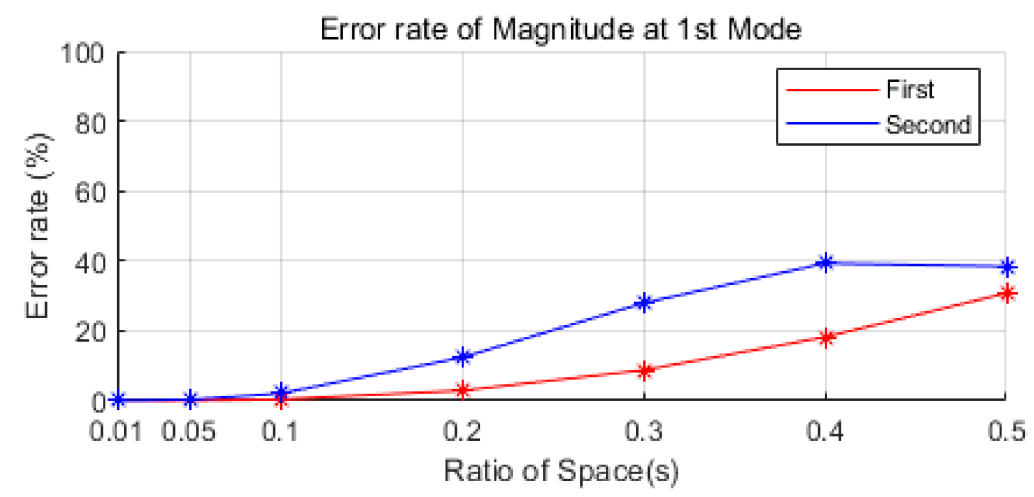

Figure 9. Error rate magnitude at the first mode.

The responses of $H_{0.01 a}$ and $H_{0.01 b}$ when $\mathrm{s} / 1=0.01$ are illustrated in Figure 10. The two response points clearly indicate the resonance and anti-resonance, and these points were well-estimated in the rotational FRF using these points. However, as illustrated in Figure 11, both response points do not exhibit resonance and anti-resonance. This is related to the shape of the mode, and because it does not indicate resonance and antiresonance, it causes a significant error in the rotational FRF estimation using this. Therefore, when selecting a response point, it is necessary to select a point where the resonance and anti-resonance modes are expressed.

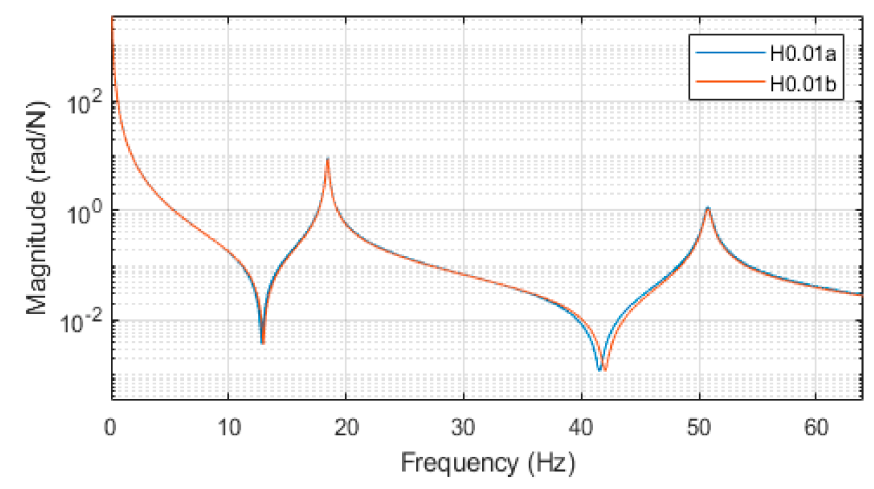

Figure 10. FRF of $H_{0.01 a}$ and $H_{0.01 b}$ at $\mathrm{s} / 1=0.01$.

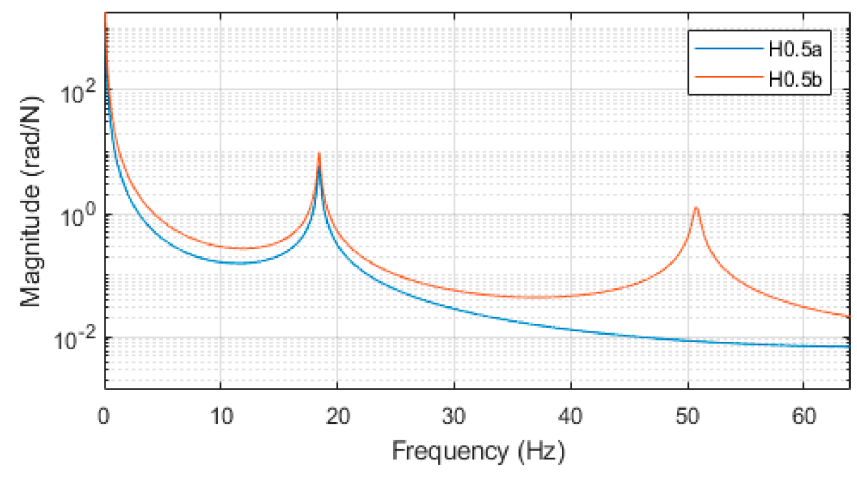

Figure 11. FRF of $H_{0.5 a}$ and $H_{0.5 b}$ at $\mathrm{s} / 1=0.5$.

\subsection{Rotaion/Moment Vibration Analysis Results}

To estimate $P_{11}$, a mode analysis should be performed. As stated in Section 2.4, mode analysis is performed on the real and imaginary parts using the peak picking method. Similar to the modal analysis of CAE, in the peak picking method, modal analysis of a sufficiently large number of modes must be performed to reduce the influence on higherorder modes. The results of the mode analysis of $L_{11}$ estimated when $H_{11}$ and s/l=0.01, 
respectively, are illustrated in Figure 12, and the reliability of the mode analysis using modal peak picking is verified. Modal analysis was performed for the first and second modes, and the results were fitted and compared with the FEM results. Although the result are similar to the FRF result of FEM, it cannot be estimated that the initial displacement becomes infinite in the real part. However, because modal analysis is performed at each natural frequency, the infinity of the initial response does not affect the modal parameter estimation.
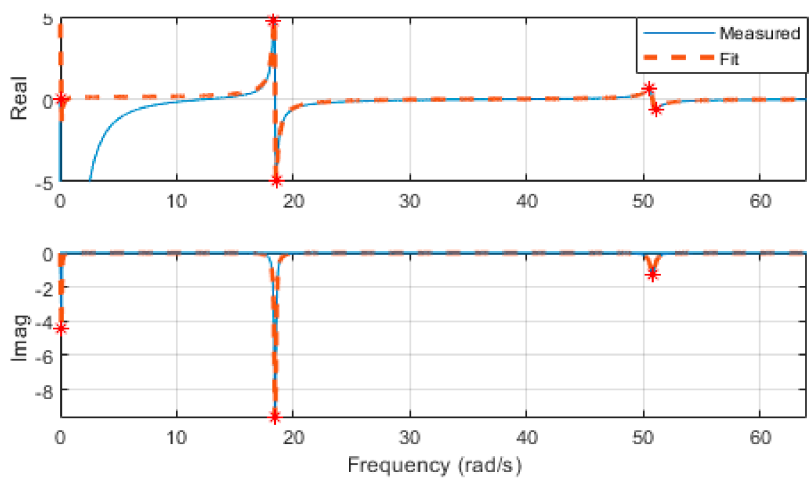

(a)
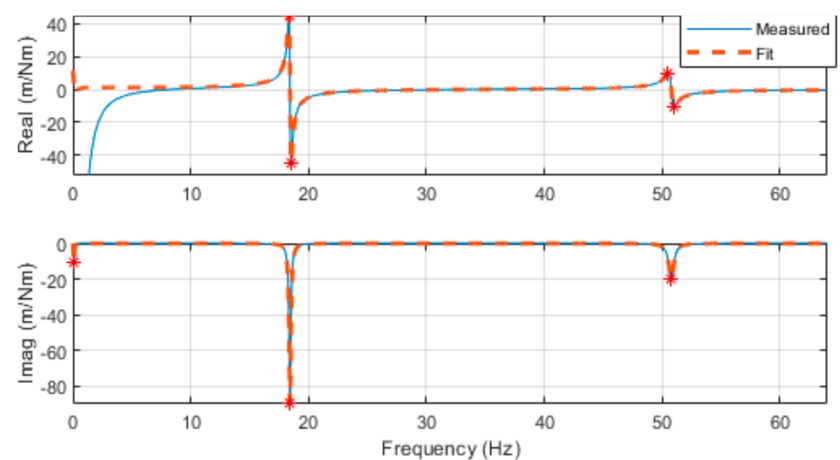

(b)

Figure 12. Modal fitting using modal peak picking; (a) is $H_{11}$ and (b) is $L_{11}$.

The estimated FRF using Schmitz's method, which is the most widely used method for estimating $P_{11}$, and the FRF using FEM is illustrated in Figure 13. Residual modes of 12.6 and $41.24 \mathrm{~Hz}$ appear between natural frequencies, which are the modes that occur, as the response of $H_{11}$ approaches zero in Equation (14). A compensation strategy was adopted to eliminate the residual modes.

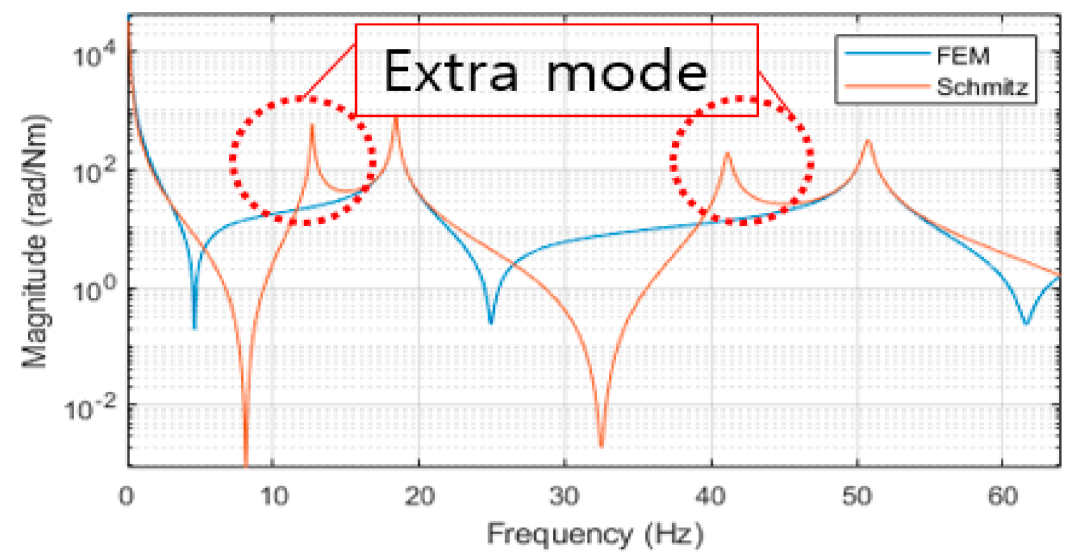

Figure 13. Comparison of $P_{11-F E M}$ with Schmitz's method.

The first compensation strategy is illustrated in Figures 14 and 15 by estimating $P_{11}$ using the modal parameters of $H_{11}$ and $L_{11}$. Figure 14 is illustrated using $L_{11}$ and $H_{11}$ by applying the first-order finite difference method according to the distance of the response point, and Figure 15 illustrates the result using the second-order finite difference method. The error increases as the distance between the response points increase, indicating a similar trend to the $L_{11}\left(=N_{11}\right)$ result. 


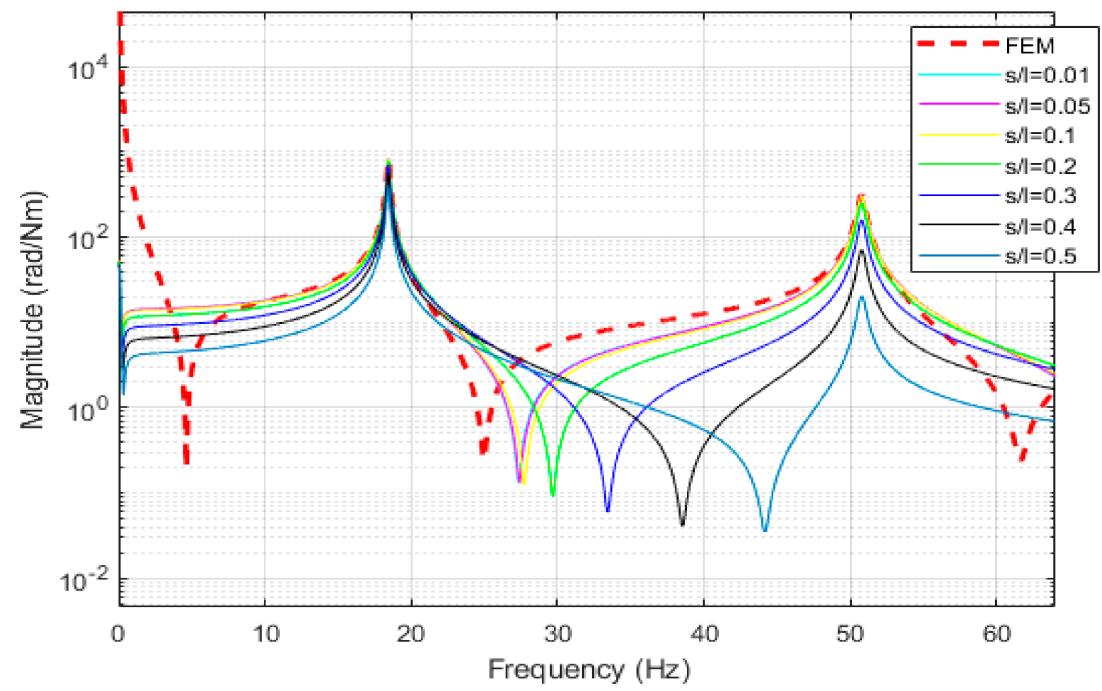

Figure 14. First compensation using the first-order finite difference technique according to $s / 1$.

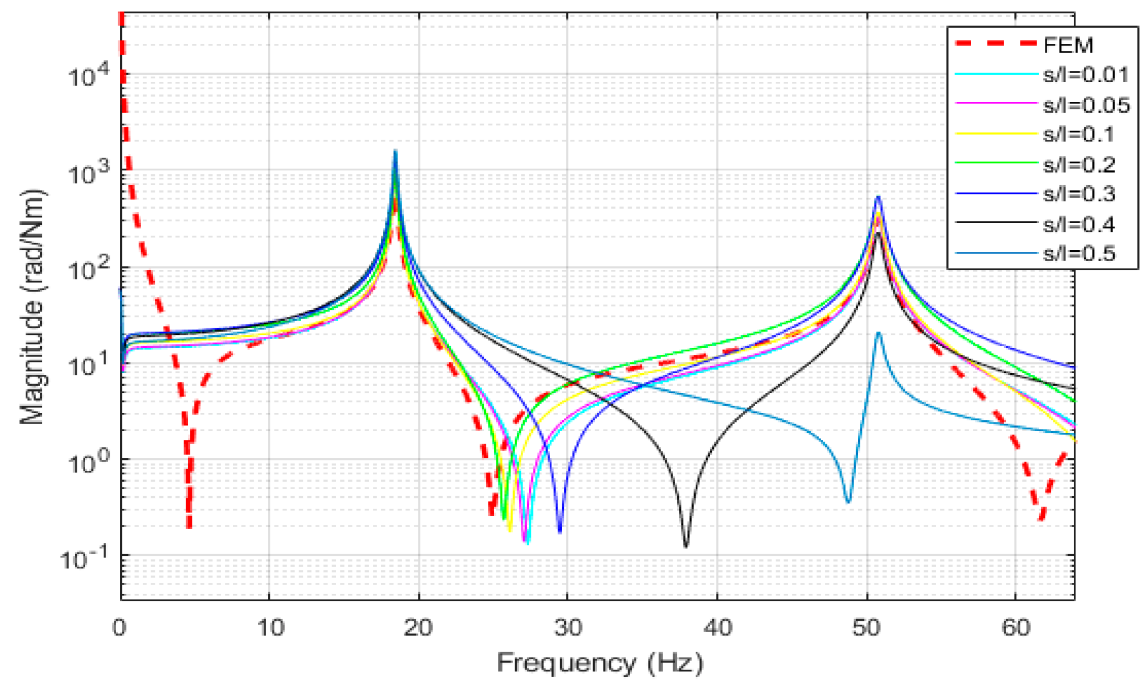

Figure 15. First compensation using the second-order finite difference technique according to s/1.

The first compensation strategy using $L_{11}$, estimated using the first-and second-order finite difference methods when $s / 1=0.01$, is illustrated in Figure 16. Because the error in estimating $L_{11}$ is negligible, a negligible error occurred in the compensation strategy as well. The accuracy of $L_{11}$ was confirmed to affect the accuracy of $P_{11}$. The first compensation strategy has a disadvantage in that it cannot express the free-free boundary condition in which the response becomes infinite at $0 \mathrm{~Hz}$. Because the boundary condition cannot be expressed, the first anti-resonance cannot be expressed as well. It would be more suitable for estimating the FRF of fixed-free or fixed-fixed boundary conditions, where the displacement response does not become infinite near $0 \mathrm{~Hz}$.

The second compensation strategy removes residual modes by performing mode analysis after calculating $P_{11}$ using Schmitz's method in Equation (14). As the result of $P_{11}$ is more accurate and $L_{11}$ is more accurate through the first compensation strategy, the second compensation strategy is performed for $\mathrm{s} / \mathrm{l}=0.01$, which has the highest accuracy of $L_{11}$. In Figure 17, Compared to the first compensation strategy, the second compensation strategy represents a free-free boundary condition in which the rotational displacement, as well as the anti-resonance, becomes infinite at $0 \mathrm{~Hz}$. The natural frequencies and magnitudes were identical. Over the second natural frequency, it appears different from the FEM result, which will reduce the error if the high-frequency mode number is sufficiently considered. 


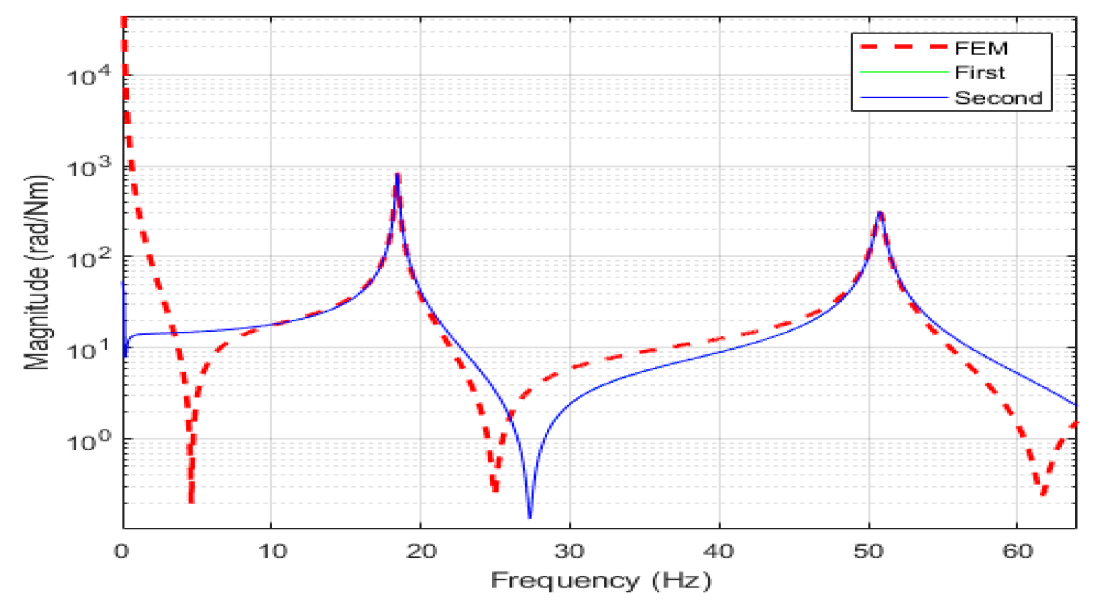

Figure 16. Error in the first compensation strategy at $\mathrm{s} / 1=0.01$.
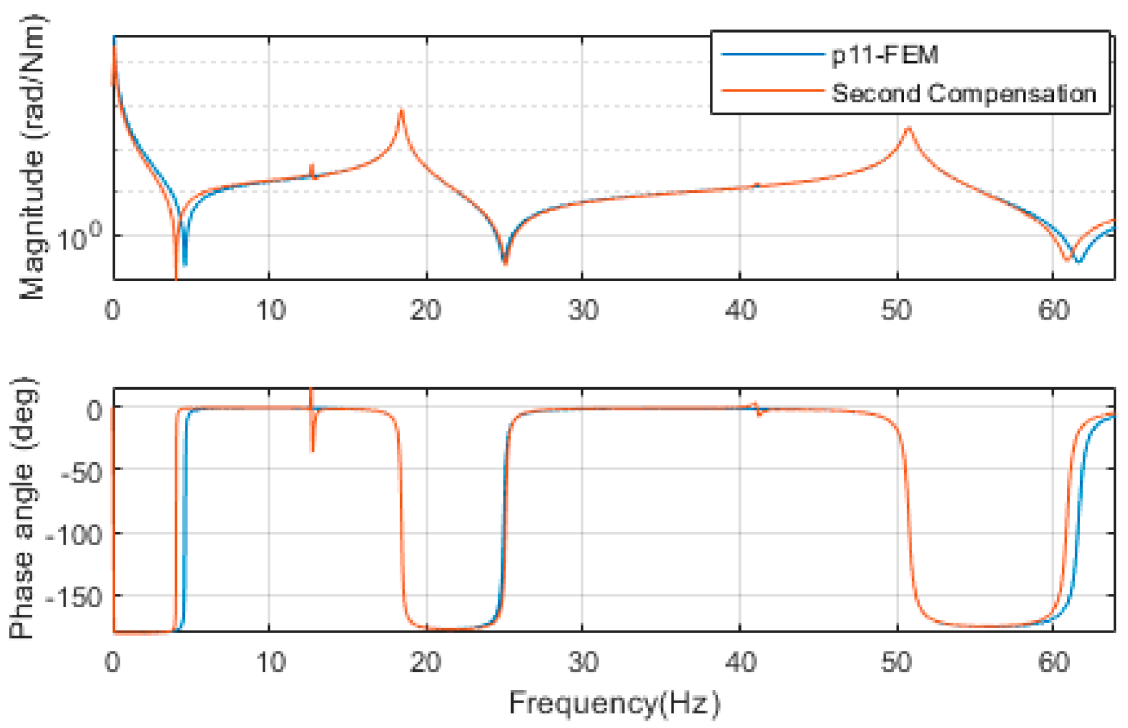

Figure 17. Second compensation strategy at $\mathrm{s} / 1=0.01$.

\section{Conclusions}

In this study, the composition of the full receptance matrix for the analysis of the dynamic characteristics of the machining equipment according to the cutting tool was determined. The full receptance matrix consists of displacement/force, displacement/moment, rotation/force, and rotation/moment receptance. Because it is difficult to obtain the receptance in the rotational direction through experiments, the receptance in the rotational direction is estimated using the receptance in the translational direction. In order to apply it to the experiment, there is no additional cost, and it is important to estimate the rotational FRF through the most accurate and relatively simple operation. Therefore, the rotational FRF was estimated as a post-processing method using the translational FRF without additional experimental equipment. In this study, the ratio of the distance of the measurement point to the length of the structure was presented in the rotation/force(=displacement/moment) FRF, and it was shown that the finite difference method was more accurate when the order was first order. In addition, the disadvantages of rotation/moment estimation of the finite difference method and Schmit's method were presented. A compensation strategy that compensates for these shortcomings was introduced. It was confirmed that the accuracy of rotation/force FRF affects the accuracy of rotation/moment FRF through the finite difference method and compensation strategy. It was shown that the free-free boundary condition could not be expressed in the first compensation strategy. The free-free boundary condition was expressed through the second compensation strategy. A compensation 
strategy selection method according to the distance of the response point and boundary conditions was presented. Vibration analysis was performed using CAE, and verification was performed using the Euler-Bernoulli beam theory.

The rotation/force (displacement/moment) receptance was estimated using the finite difference method, according to the order and distance. The number of response points varied according to the order, and the estimation accuracy varied according to the distance of the response points. In both the first-and second-order finite difference methods, the shorter the distance of the response point, the higher the accuracy; furthermore, the error increased as the order increased. Regarding the first-order finite difference method, an error of approximately $0.4 \%$ was generated in the first natural frequency at $\mathrm{s} / 1=0.1$. To estimate the displacement/moment (rotation/force) receptance in the actual test, the ratio of the distance of the response point to the total length should not exceed 0.05; even if experimental factors such as the size of the sensor are considered, the maximum ratio should not exceed 0.1 .

The rotation/moment receptance is estimated and analyzed using Schmitz's method and a compensation strategy. Schmitz's method has the disadvantage of generating residual modes other than the natural frequencies. The residual mode was removed using a compensation strategy. The first compensation strategy has a disadvantage in that it cannot estimate that the response displacement becomes infinite at $0 \mathrm{~Hz}$. The second compensation strategy estimates that the displacement of the response becomes infinite, but the error increases as the frequency increases. To reduce the error, a sufficiently large number of modes should be considered. Accurate rotation/force receptance is required to accurately estimate rotation/moment receptance. The first compensation strategy is suitable for beams with fixed ends, whereas the second compensation strategy is suitable for free-free boundary conditions.

Author Contributions: Conceptualization, J.-w.K. and J.-s.J.; methodology, J.-w.K. and J.-w.L.; software, J.-w.K., J.-h.K., S.-y.L.; validation, J.-w.K., J.-s.J., M.-s.Y., D.-y.K.; writing—original draft preparation, J.-w.K.; writing - review and editing, J.-s.J., J.-w.L. and K.-w.K.; project administration, J.-s.J. All authors have read and agreed to the published version of the manuscript.

Funding: This research received no external funding.

Institutional Review Board Statement: Not applicable.

Informed Consent Statement: Not applicable.

Data Availability Statement: Not applicable.

Acknowledgments: This research was supported by the Korea Institute of Industrial Technology (KITECH).

Conflicts of Interest: The authors declare no conflict of interest.

\section{References}

1. Schmitz, T.L.; Matthew, A.D.; Michael, D.K. Tool point frequency response prediction for high-speed machining by RCSA. J. Manuf. Sci. Eng. 2001, 123, 700-707. [CrossRef]

2. Schmitz, T.L.; Donalson, R.R. Predicting high-speed machining dynamics by substructure analysis. CIRP Ann. 2000, 49, 303-308. [CrossRef]

3. Schmitz, T.L.; Duncan, G.S. Three-component receptance coupling substructure analysis for tool point dynamics prediction. J. Manuf. Sci. Eng. 2005, 127, 781-790. [CrossRef]

4. Schmitz, T.; Burns, T. Receptance coupling for high-speed machining dynamics prediction. In Proceedings of the 21st International Modal Analysis Conference (IMAC), Kissimmee, FL, USA, 3-6 February 2003; Volume 36, pp. 19-25. Available online: https: //www.nist.gov/publications/receptance-coupling-high-speed-machining-dynamics-prediction (accessed on 12 August 2021).

5. Ewins, D.J.; Sainsbury, M.G. Mobility measurements for the vibration analysis of connected structures. J. Shock Vib. Bull. 1972, 42, 105-122.

6. Mehrpouya, M.; Graham, E.; Park, S.S. FRF based joint dynamics modeling and identification. Mech. Syst. Signal Process. 2013, 39, 265-279. [CrossRef] 
7. Lee, H.S.; Lee, H.H.; Lee, G.M. Updating of a Finite Element Model with a Damping Effect Using Frequency Response Functions. Korea Soc. Mech. Eng. 2002, 26, 872-880. [CrossRef]

8. Park, S.S.; Altintas, Y.; Movahhedy, M. Receptance Coupling for end mills. Int. J. Mach. Tools Manuf. 2003, 43, 889-896. [CrossRef]

9. Montalvão, D.; Ribeiro, A.M.; Maia, N.M.; Silva, J.M. On the estimation of rotational frequency response functions. In Proceedings of the ISMA2004 Noise and Vibration Engineering Conference, Leuven, Belgium, 20-22 September 2004; pp. $2771-2785$.

10. Chen, W.-H.; Cherng, J.-S. Modal synthesis via combined experimental and finite element techniques with consideration of rotational effects. J. Sound Vib. 1985, 103, 1-11. [CrossRef]

11. Ji, Y.; Bi, Q.; Zhang, S.; Wang, Y. A new receptance coupling substructure analysis methodology to predict tool tip dynamics. Int. J. Mach. Tools Manuf. 2018, 126, 18-26. [CrossRef]

12. Duarte, M.L.M.; Ewins, D.J. Rotational degrees of freedom for structural coupling analysis via finite-difference technique with residual compensation. Mech. Syst. Signal Process. 2000, 14, 205-227. [CrossRef]

13. Albertelli, P.; Goletti, M.; Monno, M. A new receptance coupling substructure analysis methodology to improve chatter free cutting conditions prediction. Int. J. Mach. Tools Manuf. 2013, 72, 16-24. [CrossRef]

14. Altintas, Y. Manufacturing Automation, 2nd ed.; Cambridge University Press: Cambridge, UK, 2012; pp. $109-119$.

15. Schmitz, T.L.; Scott Smith, K. Machining Dynamics; Springer International Publishing: Basel, Switzerland, 2019 ; pp. $283-337$.

16. Shmitz, T.L.; Scott Smith, K. Mechanical Vibrations: Modeling and Measurement; Springer: New York, NY, USA, 2012; pp. 199-216; 301-363.

17. Ewins, D.J. Modal Testing Theory, Practice and Application, 2nd ed.; Wiley: Hoboken, NJ, USA, 2000; pp. $260-264$.

18. Chang, K.J.; Jee, T.H.; Park, Y.P. Modal Analysis of Automotive Body Model using Mode Synthesis Method. Korean Soc. Noise Vib. Eng. (KSNVE) 1995, 34-39.

19. Jee, T.H.; Park, Y.P. Structural Dynamic Modification Using Substructure Response Function Sensitivity Method (SRFSM). Korean Soc. Noise Vib. Eng. (KSNVE) 1996, 20, 3782-3791.

20. Chang, K.J.; Jee, T.H.; Park, Y.P. Modal Synthesis Method Using Interpolated Rational DOF. Korean Soc. Noise Vib. Eng. (KSNVE) 1995, 5, 503-514. 